\section{PO 8719 INTRODUCING A NEW AFRICA MENINGITIS NETWORK - A NORTH-SOUTH COLLABORATION}

${ }^{1}$ Mosepele Mosepele*, ${ }^{2}$ Cecilia Kanyama, ${ }^{3}$ David Meya, ${ }^{4,5}$ Fiona Cresswell, ${ }^{6}$ Timothee Chammard, ${ }^{7}$ Henry Mwandumba, ${ }^{8}$ Graeme Meintjes, ${ }^{9}$ Godfrey S Mfinanga, ${ }^{10}$ Chiratidzo Ndhlovu, ${ }^{11}$ Joseph Jarvis. 'University of Botswana School of Medicine, Botswana-Harvard AIDS Institute, Botswana; ${ }^{2}$ University of North Carolina, USA; ${ }^{3}$ Infectious Disease Institute, Makerere University, Uganda; ${ }^{4}$ London School of Hygiene and Tropical Medicine, UK; ${ }^{5}$ Infectious Diseases Institute, Kampala, Uganda; ${ }^{6}$ Institute Pasteur, Paris, France: ${ }^{7}$ Malawi-Liverpool Wellcome Trust Research Programme, Lilongwe, Malawi; ${ }^{8}$ University of Cape Town, South Africa; ${ }^{9}$ National Institute of Medical Research, Dar es Salaam, Tanzania; ${ }^{10}$ University of Zimbabwe, Harare, Zimbabwe; ${ }^{11}$ London School of Hygiene and Tropical Medicine, UK, and Botswana-Harvard AIDS Institute Partnership, Botswana

\subsection{6/bmjgh-2019-EDC.165}

Background Central nervous system infections, including meningitis, continue to cause significant morbidity in Africa. HIV has contributed to the epidemiology of CNS infections in this setting. Notable advances in the study of CNS infections by several groups have demonstrated the utility of new diagnostic strategies and impact of novel treatment strategies. However, efforts to coordinate meningitis research in Africa, and between Africa and the rest of the world remain very limited.

Methods In a bid to promote a coordinated study of CNS infections across Africa, and in collaboration with other meningitis groups globally, the researchers of the AMBITION study (High Dose Ambisome on a Fluconazole Backbone for Cryptococcal Meningitis Induction Therapy in Sub-saharan Africa: A Randomised Controlled Non-inferiority Trial) are leveraging the EDCTP support for the AMBITION trial to set up an Africa Meningitis Trials Network.

Results The Africa Meningitis Trials Network (AMNET) was launched in Malawi in early 2018. Main achievements since the launch of the network, include an internal review of meningitis research across network sites and launch of the network website. The network also has two study protocols pending ethics review at all sites. These studies will provide much needed information on resources available for meningitis care, research and provide a baseline epidemiology of meningitis in Africa.

Conclusion AMNET provides a rare opportunity for investigators interested in meningitis research to leverage the ongoing AMBITION trial to conduct Africa-wide preliminary research on meningitis. The network is recruiting additional members in Africa and globally to collaborate on meningitis research, and also apply for research funding to support meningitis work. Anyone interested in knowing more about the network should contact the AMNET communications officer, Ms Phum'lani Machao, phumlani.machao@gmail.com

\section{PO 8720 IMPACT OF EXPANDED USE OF AN ONLINE ETHICS REVIEW SYSTEM IN AFRICA: A PARTNERSHIP BETWEEN IAVI, COHRED, AND EDCTP WITH NATIONAL REGULATORY AGENCIES}

${ }^{1}$ Francis Kombe*, ${ }^{2}$ Marzelle Haskins, ${ }^{3}$ Amina Mkonje Salim, ${ }^{4}$ Flavia Ayebazibwe, ${ }^{1}$ Carel IJsselmuiden. 'Council on Health Research for Development (COHRED), Geneva, Switzerland; ${ }^{2}$ Pharma Ethics, South Africa; ${ }^{3}$ Strathmore University, Nairobi, Kenya; ${ }^{4}$ Uganda Virus Research Institute (UVRI), Entebbe, Uganda

\subsection{6/bmjgh-2019-EDC.166}

Background RHInnO Ethics is a cloud-based online ethics review system that enables Reseach Ethics Committees (RECs) to receive, review and approve applications online. Over the last five years, RHInnO Ethics has been installed in 40 RECs that operate in 12 African countries.

Methods Pharma Ethics (South Africa), Strathmore University (Kenya) and Uganda Virus Research Institute-UVRI (Uganda) started using RHInnO Ethics in 2014, 2015 and 2017 respectively. Although the platform is currently installed in 40 RECs in Africa, only 1 systematic evaluation has been done to objectively assess its impact on the quality and efficiency of ethics review. Here, we present the experiences of using RHInnO Ethics from three RECs situated in East Africa (2 RECs) and South Africa (1 REC).

Results Strathmore University started using the online ethics review system since the beginning of its operation, while Pharma Ethics and UVRI transitioned from a manual to an electronic system. Benefits associated with the use of the platform include improved efficiency in communication among key players of the ethics review processes, improved quality of review through standardisation and harmonisation of ethics review procedures, easy management of information, increased submissions and improved turnaround time. The progress of review can also be tracked any time by all users. Challenges include change management from the manual to the online system, difficulties in learning new versions of the system and training new reviewers not conversant with the system, interruption of internet access and limited ability to personalise the platform.

Conclusion There is a need to get buy-in from regulatory authorities to enforce the adaption of the system to all RECs in order to make research coordination in Africa easier. RHInnO Ethics has been shown to improve efficiency and quality of reviews. However, the transition to and full adoption of the online system continues to be slow. To accelerate and scale up the adoption of RHInnO Ethics, there is a need for developers, RECs and sponsors of RECs to engage with REC regulatory authorities, and identify responsive models of financing and funding RECs in order to increase efficiency and quality of ethics review in sub-Saharan Africa. 